\title{
Terahertz optical and mechanical properties of the gelatin- starch-glycerol-bentonite biopolymers
}

\author{
Tianmiao Zhang ${ }^{1 *}$, Maria Zakharova ${ }^{2}$, Anna Vozianova ${ }^{1}$, Aleksandr Podshivalov ${ }^{2}$, Maria Fokina ${ }^{2}$, \\ Ravshanjon Nazarov ${ }^{1}$, Anna Kuzikova ${ }^{1}$, Petr Demchenko ${ }^{1}$, Mayya Uspenskaya ${ }^{2}$, \\ and Mikhail Khodzitsky ${ }^{1}$ \\ ${ }^{1}$ Terahertz Biomedicine Laboratory, ITMO University, Saint Petersburg 199034, Russia \\ ${ }^{2}$ International Scientific and Research Institute of Bioengineering, ITMO University, Saint Petersburg 197101, Russia \\ * e-mail: tmzhang91@gmail.com
}

\begin{abstract}
With the fast development of terahertz technology in medical diagnosis and monitoring, it has become important to investigate the application of $\mathrm{THz}$ radiation in the cancer treatment assessment during the therapy. In this paper, a buccal drug delivery system is studied as the first step towards this application. The drug delivery system is based on a gelatin-starch biopolymer matrix filled with plasticizing glycerol and various contents of reinforcing particles of bentonite clay. The biopolymers were subjected to morphology analysis using optical microscopy, analysis of mechanical tensile properties, and analysis of terahertz optical properties, followed by a theoretical approach of the experiment. The results show a visible effect of the bentonite content on both of the mechanical and terahertz optical properties of the biopolymer. These findings allow us to confirm the feasibility of using $\mathrm{THz}$ radiation for cancer assessment during therapies. The proposed biopolymer also has the potential to be applied as a substrate when carrying out in-vivo optical property measurement of biotissue in terahertz frequency range. (C) 2020 Journal of Biomedical Photonics \& Engineering.
\end{abstract}

Keywords: biopolymer; terahertz time-domain spectroscopy; effective medium theory; bentonite; drug delivery system; mechanical properties; optical properties.

Paper \#3359 received 1 Apr 2020; revised manuscript received 28 May 2020; accepted for publication 15 Jun 2020; published online 22 Jun 2020. doi: 10.18287/JBPE20.06.020304.

\section{Introduction}

Terahertz (THz) radiation is attracting scientists' attention around the world because it has been considered as an efficient non-destructive and nonionizing optical method for biological object investigation. For example, $\mathrm{THz}$ imaging has been previously shown to be capable of distinguishing normal (healthy) tissue from its cancerous form [1-4]. Wound assessment of skin burn using $\mathrm{THz}$ radiation was also investigated [5]. The THz optical properties of oral tissue and its cancerous form have been measured and potential diagnosis method using $\mathrm{THz}$ radiation was given [6]. A $\mathrm{THz}$ endoscope system was described in article [7], which made in vivo cancer diagnosis possible.
The following potential research topic is to investigate the application of $\mathrm{THz}$ radiation in the cancer treatment assessment during the therapy. Applying drug delivery system made by biocompatible/biodegradable polymers (biopolymers) on the surface of cancerous area is a standard treatment protocol of oral and skin cancers $[8,9]$. $\mathrm{THz}$ radiation needs to penetrate through the biopolymer for the treatment assessment. However, no comprehensive investigation of drug delivery system in the $\mathrm{THz}$ frequency range has been done. Its $\mathrm{THz}$ optical properties, i.e. the $\mathrm{THz}$ waves propagation inside of drug delivery systems, are still unknown.

The drug delivery system based on gelatin-starch biopolymers is chosen as the research object, due to its attractive properties, such as, good solubility in water, 
processability by melt methods, high film-forming properties, relatively low cost and availability on the world market $[10,11]$. The solid-state materials, which obtained from gelatin-starch systems have such properties as non-toxicity, edibility and digestibility in organisms, biodegradability, higher mechanical properties and vapor-gas barrier properties compared to pure materials [12]. The use of gelatin-starch systems in the shell coat of the micro-capsules for drug delivery $[13,14]$, soft and hard capsule materials for drugs [15], edible films and coatings for extending the shelf life of food products $[12,16]$ and orally disintegrating films and carriers embedded vitamins and drugs for buccal drug delivery $[17,18]$ has been reported in the recent studies.

This work aims to study the $\mathrm{THz}$ optical properties of gelatin-starch biopolymers and to evaluate the feasibility of using $\mathrm{THz}$ radiation as a medical assessment technique during cancer therapy. We prepared biopolymers based on gelatin-starch-glycerol composition with different content of the bentonite reinforcing particles and various thicknesses. The structural analysis of morphology of the resulting biopolymers was studied using light microscopy and mechanical tensile tests. The refractive index and the absorption coefficient of the biopolymers were studied in the frequency range of $0.2-1 \mathrm{THz}$ using $\mathrm{THz}$ timedomain spectroscopy (TDS). Three mathematical models known as Complex Refractive Index (CRI), Landau-Lifshitz-Looyenga (LLL) and Bruggeman models, and additionally an iterative method based on Bruggeman model were used to seek for the best mathematical approach to describe the proposed biopolymer. The THz optical properties of the proposed biopolymers were also compared with those of healthy and cancerous oral tissues for the feasibility evaluation of $\mathrm{THz}$ medical assessment technique during cancer therapy.

\section{Materials and methods}

\subsection{Materials}

Potato starch is from local source (analytical grade) in the form of white powder and food grade gelatin is from local source in form of light-yellow grains were obtained by Vekton (Russia). Glycerol in off-white liquid form is provided by LenReactiv (Russia). Hydrophilic bentonite particles "Nanoclay" with mean particle size of $21 \mu \mathrm{m}$ (CAS \# 1302-78-9) was obtained by Sigma Aldrich (USA).

\subsection{Biopolymer preparation}

The biopolymers were prepared by casting gel-forming solution and subsequent drying. At first, the single water-biopolymer solution of gelatin and starch with $5 \mathrm{wt} . \%$ concentration of dry basis were prepared as the biopolymer matrix. Both gelatin and starch were swollen separately in distilled water in $10 \mathrm{~min}$. Then the solutions were dissolved and homogenized at $60{ }^{\circ} \mathrm{C}$ and at $90{ }^{\circ} \mathrm{C}$ with continuous stirring for gelatin and starch solution, respectively. After complete homogenization ( $\approx 10$ minutes), the single biopolymer solution of gelatin and starch was blended with the volume ratio of 90 vol. $\%$ and 10 vol. $\%$, respectively. Afterwards, the solution was continuously being stirred for $5 \mathrm{~min}$ at $70{ }^{\circ} \mathrm{C}$.

Next, the water and filler suspensions were prepared. The glycerol in count of $30 \mathrm{wt} . \%$ from dry basis of main biopolymers was dissolved in distilled water with continuous stirring for $3 \mathrm{~min}$ at room temperature. Then bentonite particles with various contents were added to the water-glycerol solution. Then the obtained filler suspensions were homogenized at $70{ }^{\circ} \mathrm{C}$ for $10 \mathrm{~min}$ with continuous stirring.

After the preparation of the blended biopolymer solutions and filler suspensions, they were mixed and homogenized at $70{ }^{\circ} \mathrm{C}$ for $5 \mathrm{~min}$ with continuous stirring. The obtained solutions were additionally sonicated using standard ultrasonic homogenizer at $70{ }^{\circ} \mathrm{C}$ for $15 \mathrm{~min}$. The final gel-forming solutions were casted into polystyrene Petri dishes in various volumes for the material preparation with different thicknesses. Casted solutions were dried using the drying oven UT-4620 Ulab (Russia) equipped with bubble level holder at $26 \pm 2{ }^{\circ} \mathrm{C}$ for $48 \mathrm{~h}$.

After complete fabrication of the biopolymers with different thicknesses, their moisture contents were determined using the standard gravimetric method. Based on the concentrations of the components in the solutions and the moisture contents of dried biocomposites, the volume fractions of all components in the fabricated biopolymers were determined depending on $\varphi$ and presented in Table 1.

Table 1 The volume fractions of the components in the biopolymers depending on $\varphi$.

\begin{tabular}{ccccccc}
\hline$\varphi$ & Gelatin & $\begin{array}{r}\text { Potato } \\
\text { starch }\end{array}$ & \multicolumn{2}{c}{ Glycerol Bentonite } & Water \\
(wt.\%) & (vol.\%) & $\begin{array}{c}\text { (vol.\%) } \\
\text { (vol.\%) }\end{array}$ & (vol.\%) & (vol.\%) \\
\hline 0 & 74.0 & 7.4 & 8.6 & 0 & $10 \pm 2$ \\
3 & 72.0 & 7.2 & 8.1 & 2.7 & $10 \pm 2$ \\
5 & 70.6 & 7.0 & 7.9 & 4.5 & $10 \pm 2$ \\
7 & 69.0 & 6.9 & 7.8 & 6.3 & $10 \pm 2$ \\
9 & 68.0 & 6.8 & 7.7 & 7.5 & $10 \pm 2$ \\
\hline
\end{tabular}

For additional mechanical tests, biopolymers were prepared with an extended range of $\varphi$ from 1 to $11 \mathrm{wt} . \%$ with increment of $2 \mathrm{wt} \% \%$ from dry basis of main biopolymer matrix. 


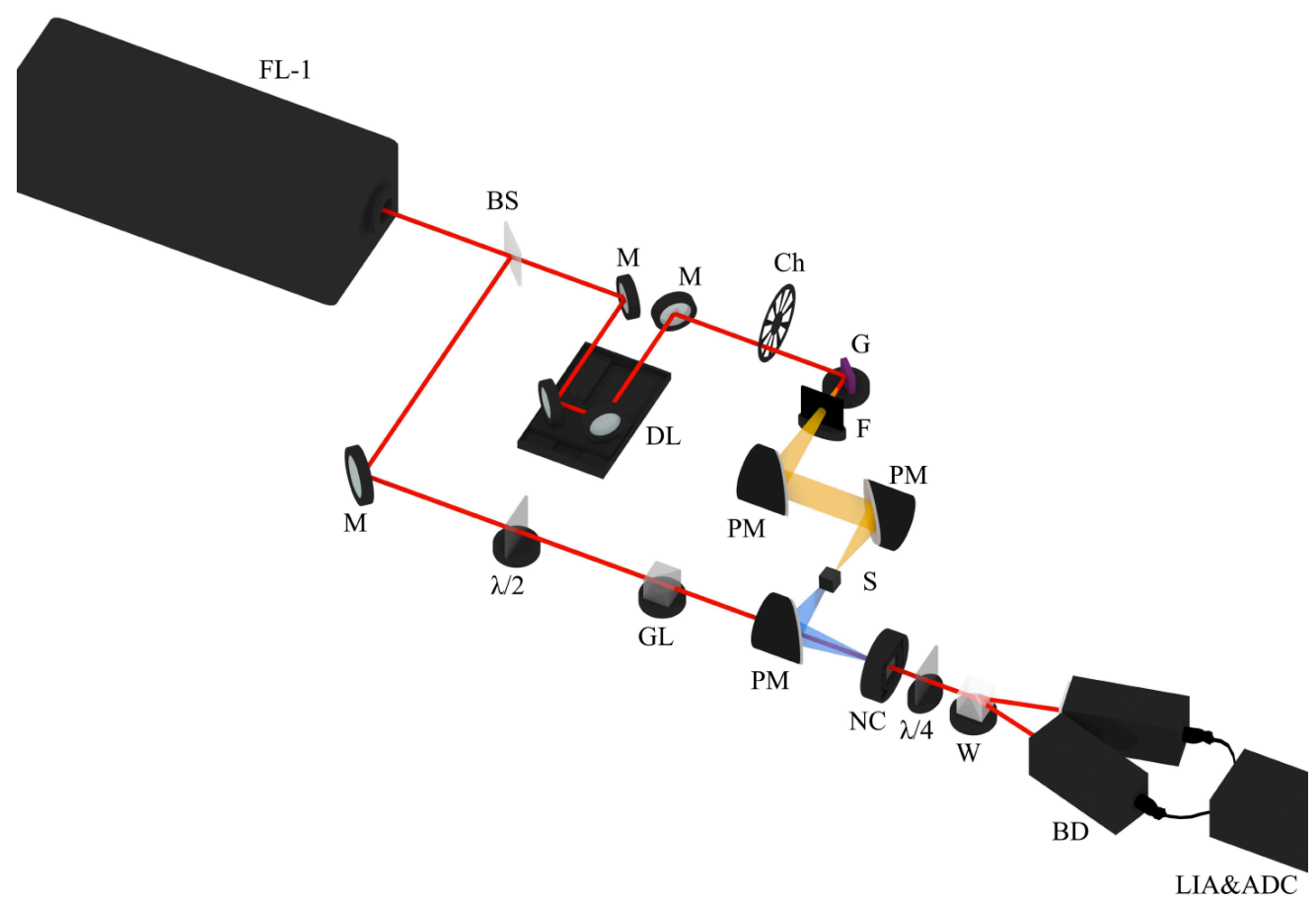

Fig. 1 Scheme of THz time-domain spectrometer (TDS). FL-1 - femtosecond IR laser, M - mirror, BS - beam splitter, $\lambda / 2$ - half-wave plate, GL - Glan prism, DL - optical delay line, CH - chopper, G - THz-generator (InAs crystal locates inside of the constant magnetic field of $2 \mathrm{~T}$ ), $\mathrm{F}$ - Teflon filter, PM - parabolic mirror, $\mathrm{S}$ - sample, NC - nonlinear crystal detector (CdTe), $\lambda / 4$ - quarter-wave plate, W - Wollaston prism, $\mathrm{BD}$ - balanced photodiodes, LIA - Lock-in-amplifier, ADC - analog-to-digital converter.

\subsection{Investigation methods}

Morphology and mechanical properties. The thickness of samples $(d)$ was determined at ten different points on each composition using a standard digital micrometer T050011 Tehrim (Russia) with an accuracy of $\pm 5 \mu \mathrm{m}$. Tensile properties of the samples, obtained from $15 \mathrm{ml}$ volume of solutions, were determined using the electromechanical testing machine Instron 5966 (USA) equipped with a $10 \mathrm{kN}$ load cell, the pneumatic action grip system and special software Bluehill 3 . During the tests 6 film samples of each composition with rectangular shape and dimension of $50 \times 10 \mathrm{~mm}^{2}$ were subjected to tensile deformation at $50 \mathrm{~mm} / \mathrm{min}$ rate that conforms to the standard ASTM D882-12. To obtain the micro-transmitted light images of the biopolymer structures, we used an Olympus STM6 microscope.

Terahertz time-domain spectroscopy. Optical properties and spectral characteristics of the samples were measured using the THz-TDS (Fig. 1). The THz signals were recorded using the electro-optical sampling technique.

First, infrared (IR) femtosecond laser beam is divided into a pump beam and a probe beam at 90:10 ratio intensity of beam source, respectively. The pump beam passes through optical delay line (motorized reflector), optical chopper and incidents on indium arsenide (InAs) crystal positioned in the constant magnetic field of $2 \mathrm{~T}$. The generation of terahertz radiation using the InAs crystal is based on the Dember effect. Next, $\mathrm{THz}$ beam is focused, passes through sample and then incidents on the cadmium tellurium crystal (NC). Meanwhile, the probe beam passes through a half-wavelength plate and a Glan prism to achieve the linear polarization. After that the probe beam also enters in NC through a hole in the parabolic mirror and focusing lens. The CdTe crystal turns to be anisotropic due to the Pockel's effect when there is incidence of $\mathrm{THz}$ radiation, and the polarization of the probe beam becomes elliptical. Afterwards, the probe beam passes the quarter-wave plate and the Wollaston prism, after which splits into two orthogonal components (vertical and horizontal polarization). These beams incident on the balanced detector, and it gives analogue signal of the intensity differences between these two polarizations. This difference is proportional to the $\mathrm{THz}$ beam amplitude. Then the analogue signal goes into lock-in-amplifier as input, while reference input is given from the chopper. After that result signal gets into analog-to-digital converter (ADC) and then digital signal enters PC. The arrival time of the probe pulse with respect to the $\mathrm{THz}$ pulse is varied by moving the optical delay line, and the $\mathrm{THz}$ pulse is scanned and its waveform is finally registered. The optical delay line is controlled by PC through a digital-to-analog converter (DAC).

The THz-TDS has the following parameters: the wavelength of femtosecond laser is $1040 \mathrm{~nm}$, the pulse duration $\leq 200 \mathrm{fs}$, the average power is up to $1 \mathrm{~W}$; the terahertz radiation output: the pulse duration is around $2.7 \mathrm{ps}$, the frequency of modulation is $773 \mathrm{~Hz}$, the 
frequency range is from 0.2 to $1 \mathrm{THz}$, the dynamic range is up to $50 \mathrm{~dB}$. In the experiments, the frequency resolution is approximately $50 \mathrm{GHz}$.

Extraction of optical properties. The optical property measurement of biopolymers was performed in transmission mode. First, the reference signal was obtained while no sample was placed in the TDS setup. Then the samples were placed on the sample mount and were measured at ambient temperature $\left(20^{\circ} \mathrm{C}\right)$ one by one. For each sample, five measurements were performed continuously. Then the averaged waveforms were used to calculate the optical properties of the samples to diminish the system errors. The waveforms were first filtered using the Gaussian window to acquired correct phases [19]. After the filtration, the Fourier transform was done to extract information such as amplitude and phase. The following formulas were then used to calculate the refractive index and absorption coefficient getting from the transmission mode [20-22]:

$$
\begin{gathered}
\alpha(v)=-\frac{2}{d} \ln \frac{\left|\hat{E}_{\text {sample }}(v)\right|}{\sqrt{T(v)}\left|\hat{E}_{\text {reference }}(v)\right|} ; \\
n(v)=1+\frac{c\left[\psi_{\text {sample }}(v)-\psi_{\text {reference }}(v)\right]}{2 \pi v d} ; \\
T(v)=1-R=1-[n(v)-1]^{2} /[n(v)+1]^{2},
\end{gathered}
$$

where $\alpha$ - the absorption coefficient of the sample, $n$ - the refractive index of the sample, $\hat{E}_{\text {reference }}$, $\hat{E}_{\text {sample }}$ - the complex amplitudes of reference and sample signal, respectively, $\psi_{\text {reference }}, \psi_{\text {sample }}-$ the phase of reference and sample signal, respectively, $v-$ the frequency, $d$ - the thickness of the sample, $R$ - the Fresnel loss (reflectance) at the air-sample interface, $c-$ the speed of light.

The optical property measurement of each component was carried out in reflection mode. One component was mounted on a plastic plate, was covered by a dielectric lossless silicon window with the thickness of $325 \mu \mathrm{m}$ and the refractive index of 3.425 . The $\mathrm{THz}$ beam reached the surface of window at normal incidence, then the $\mathrm{THz}$ beam was reflected at airwindow interface and window-sample interface. Thus in one measurement two pulses were recorded. The first pulse is the reference and the second pulse is the sample signal. The two pulses were extracted using Gaussian windows accordingly. The same as the measurement of biopolymers, for each component five measurements were carried out to calculate the average amplitude of the $\mathrm{THz}$ waveform. For the extraction of the optical properties getting from the reflection mode, the following formulas were used [23]:

$$
\begin{gathered}
n_{S}(v)=\frac{n_{w}\left(1-A^{2}\right)}{1+A^{2}+2 A \cos \psi} \\
\alpha_{S}(v)=\frac{2 n_{w} A \sin \psi}{1+A^{2}+2 A \cos \psi} \cdot \frac{4 \pi}{c}
\end{gathered}
$$

$$
\begin{aligned}
& A \exp (i \psi)= \\
& =\frac{1-n_{w}^{2}}{4 n_{w}} \exp \left[2 j n_{w} \frac{2 \pi v d_{w}}{c}\right] \frac{\hat{E}_{\text {sample }}(v)}{\hat{E}_{\text {reference }}(v)}
\end{aligned}
$$

where $n_{s}, n_{w}$ - the refractive index of the sample and dielectric window, respectively, $\alpha_{S}-$ the absorption coefficient of the sample, $v-$ the frequency, $\hat{E}_{\text {reference }}$, $\hat{E}_{\text {sample }}$ - the complex amplitudes of reference and sample signal, respectively, $A$ and $\psi$ - the amplitude and the phase of complex expression from Eq. (6), $d_{w}$ - the thickness of the window, $c$ - the speed of light.

\section{Experiment results}

\subsection{Morphology of biopolymers}

Fig. 2 shows the typical morphology of the gelatinstarch-glycerol biopolymer with a phase separation of the components in the form of a continuous major phase of gelatin and a minor starch microdomain phase built into it. Also, microphotographs show dark regions localized mainly close to the interfacial region of biopolymers. These objects belong to the dispersed phase of bentonite filler particles, which absorb visible light. The microphotograph in Fig. $2 b$ shows that an increase in $\varphi$ to $7 \mathrm{wt} \% \%$ leads to an increase in the planar area of the dark regions associated with the filler phase. In this case, the localization of the filler is observed more clearly.

\subsection{Mechanical properties of biopolymers}

Fig. 3 presents the results of mechanical tensile testing of the biopolymers. The particles of bentonite clay in an amount of $\varphi<5$ wt. $\%$ do not significantly change the tensile strength of the materials. However, a further increase in the bentonite content to $\varphi=7 \mathrm{wt} . \%$ leads to a twofold (to $\sigma=19 \mathrm{MPa}$ ) reinforcement of the composite polymer matrix. The results in Fig. $3 \mathrm{~b}$ show that the elongation at break $(\eta)$ of the biopolymers decreases from $\eta=81 \%$ to $\eta=51.5 \%$ as the concentration of bentonite particles increases from $\varphi=1$ wt. $\%$ to $\varphi=9$ wt. $\%$. Besides, the subsequent increase of the $\varphi$ value does not affect the flexibility of the material. As can be seen from Fig. 3c, the stiffness of the biopolymer matrix increases significantly only from $E=140 \mathrm{MPa}$ at $\varphi=7 \mathrm{wt} . \%$ to $E=447 \mathrm{MPa}$ at $\varphi=9$ wt. $\%$. 


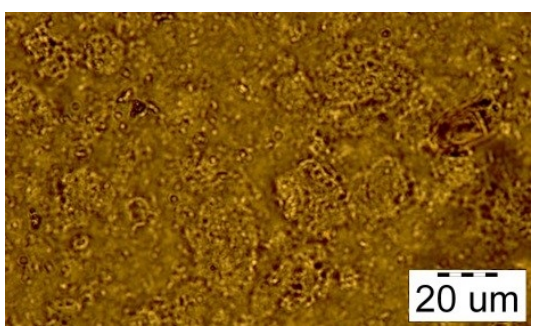

(a)

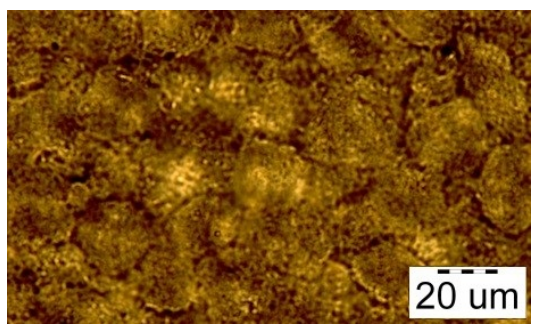

(b)

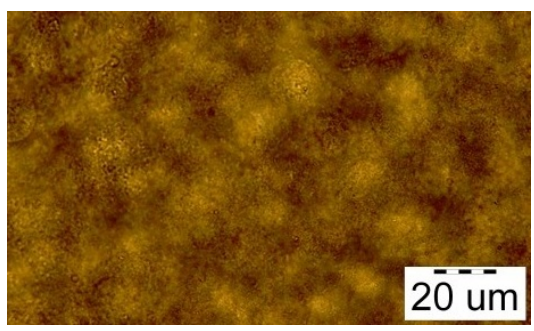

(c)

Fig. 2 Optical transmitted-light microphotographs of the biopolymers at (a) $\varphi=3$ wt.\%, (b) $\varphi=7$ wt. $\%$ and (c) $\varphi=9$ wt. $\%$.

\subsection{THz optical properties of biopolymers}

First, the biopolymers with the same bentonite content of $\varphi=9$ wt. $\%$ but with the different thicknesses were fabricated and measured. As seen in Fig. 4a, the refractive indices of the biopolymers becomes more reproducible in $0.2-1 \mathrm{THz}$ frequency range as the thickness of the biopolymer increases over $186 \mu \mathrm{m}$. A resonance can be observed when biopolymer is thin. The absorption coefficient (Fig. 4b) in the frequency range from 0.2 to $1 \mathrm{THz}$ has the same behavior, that by increasing the biopolymer thickness, the absorption coefficient becomes more reproducible.

In general, the $\mathrm{THz}$ optical properties of a material should not change no matter how its thickness changes. But in our case, the properties change because the proposed biopolymer with the thicknesses lower than $186 \mu \mathrm{m}$ is inhomogeneous in the direction of $\mathrm{THz}$ wave propagation.

The biopolymers without bentonite were also measured to investigate the influence of bentonite concentration on the $\mathrm{THz}$ optical properties of the biopolymers. The results (Fig. 5) show that with an increase of bentonite content, the refractive index increases, while the absorption coefficient has no clear dependency on bentonite concentration.

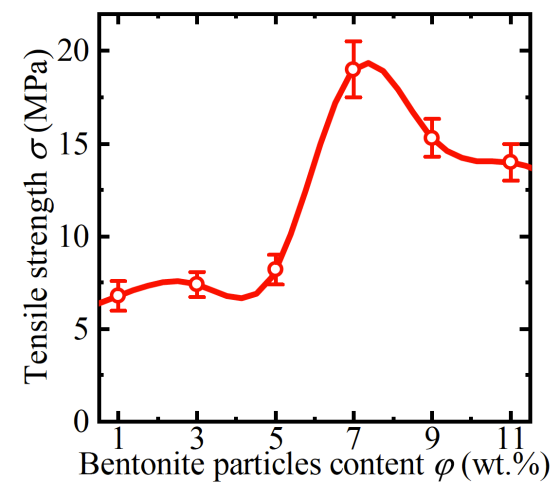

(a)

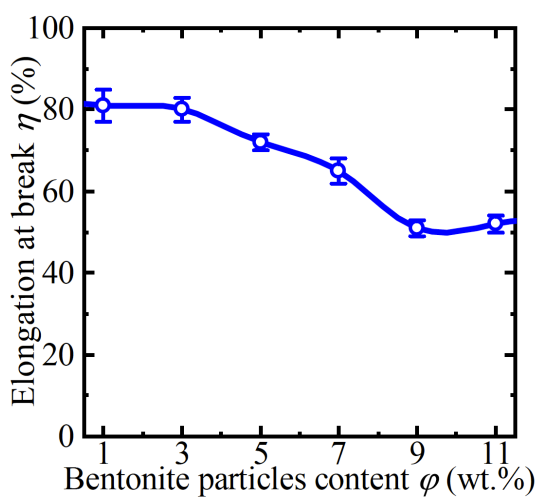

(b)

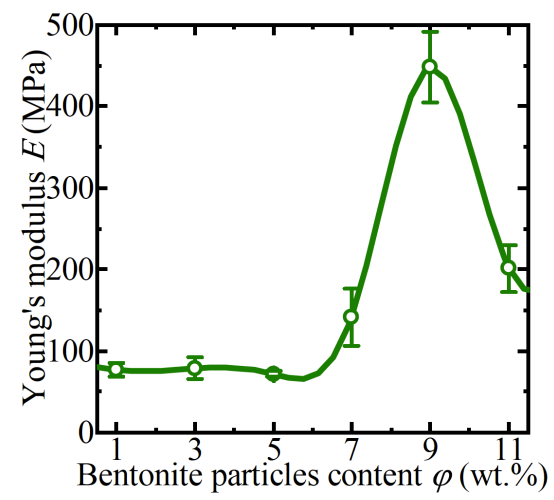

(c)

Fig. 3 Behavior of the (a) tensile strength, (b) elongation at break and (c) Young's modulus of the biopolymers depending on $\varphi$ value.

To study further, more biopolymers with the thicknesses less than $186 \mu \mathrm{m}$ were also measured in the frequency range from 0.2 to $1 \mathrm{THz}$. Control variates method was used. First, the thickness of the samples was controlled as $0.125 \mathrm{~mm}$. In Fig. 6a, the refractive indices of the biopolymers increase monotonically as the $\varphi$ value increases. The absorption coefficients have no clear dependency on bentonite concentration. The results indicate that the influence of bentonite content on the $\mathrm{THz}$ optical properties of biopolymers thicker than $186 \mu \mathrm{m}$ also happens with the biopolymer thinner than $186 \mu \mathrm{m}$. The homogeneity of the biopolymer does not change this behavior. 


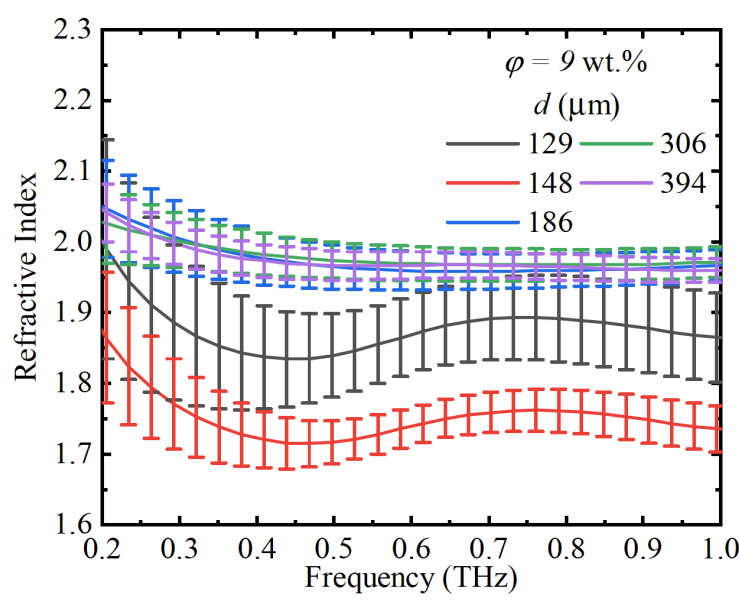

(a)

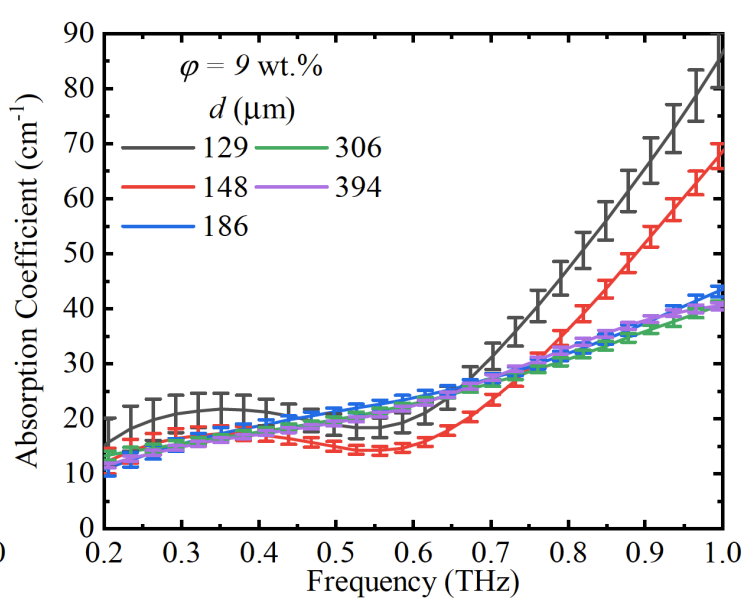

(b)

Fig. 4 The dispersion of (a) refractive index and (b) absorption coefficient of the biopolymers at $\varphi=9$ wt. $\%$ and different thicknesses in the frequency range of $0.2-1.0 \mathrm{THz}$.

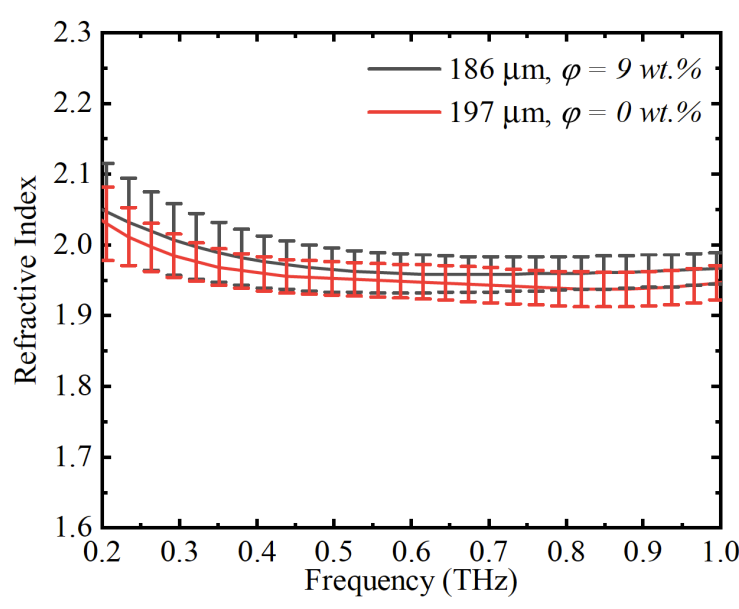

(a)

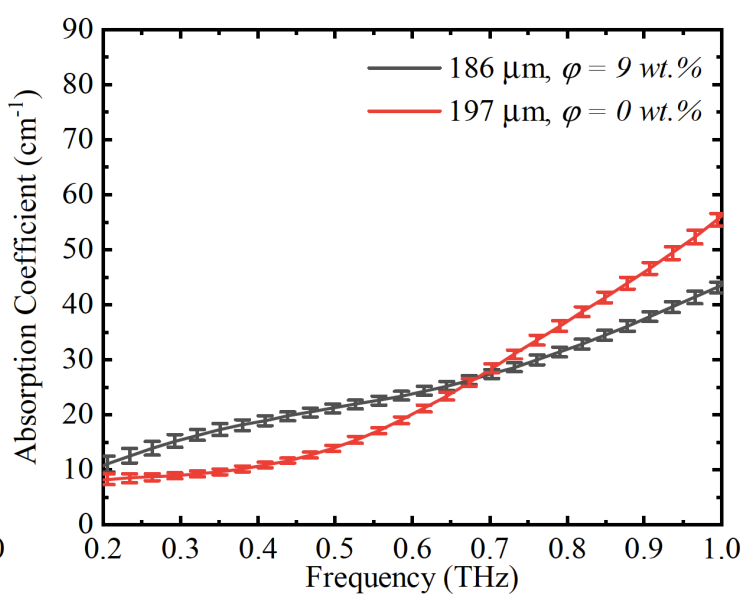

(b)

Fig. 5 The dispersion of (a) refractive index and (b) absorption coefficient of the biopolymers at $\varphi=9$ wt. $\%$ and $\varphi=0$ wt. $\%$ with the similar thicknesses of $186 \mu \mathrm{m}$ and $197 \mu \mathrm{m}$, respectively, in the frequency range of $0.2-1.0 \mathrm{THz}$.

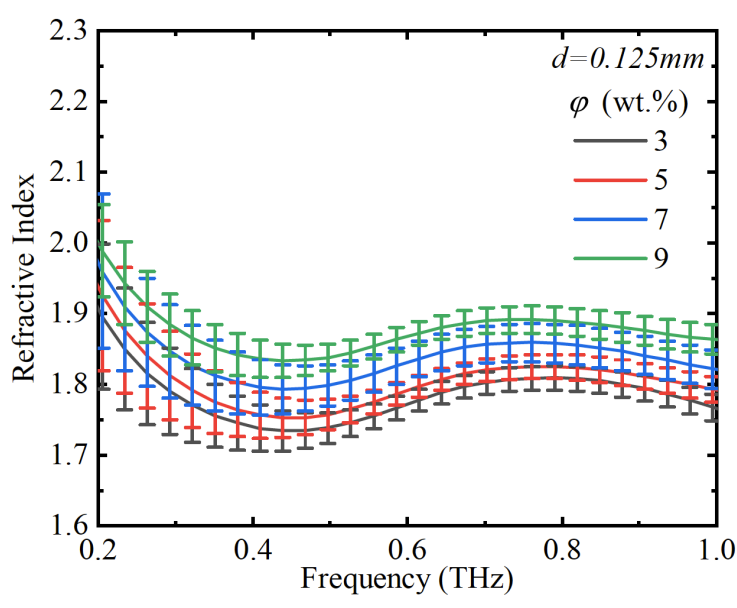

(a)

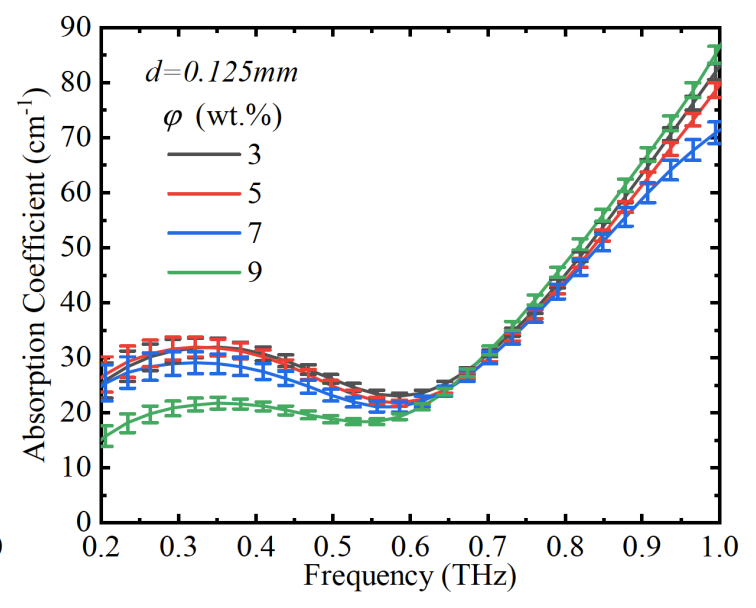

(b)

Fig. 6 The dispersion of (a) refractive index and (b) absorption coefficient of the biopolymers at various $\varphi$ value and the fixed thickness of $125 \mu \mathrm{m}$ in the frequency range of $0.2-1.0 \mathrm{THz}$. 


\subsection{THz optical properties of components}

$\mathrm{THz}$ optical properties of each components were also measured using THz TDS in reflection mode as shown in Fig. 7. THz optical properties of water were also compared with the published data [24] for the device calibration. The data of each components were then used in the numerical models.

\section{Numerical models}

Generally, the interaction of terahertz waves with a composite material is described by effective medium theory (EMT), which can be used to estimate the complex permittivity $\hat{\varepsilon}_{R}$ of a composite material. In this paper, three well-known theoretical models of EMT, namely CRI model [25], LLL model [25] and Bruggeman model [26] generalized for the anisotropic inclusions of $\mathrm{N}$-component mixture, as well as an iterative method [27] based on Bruggeman model were used for the optical property prediction of 5-component mixture.

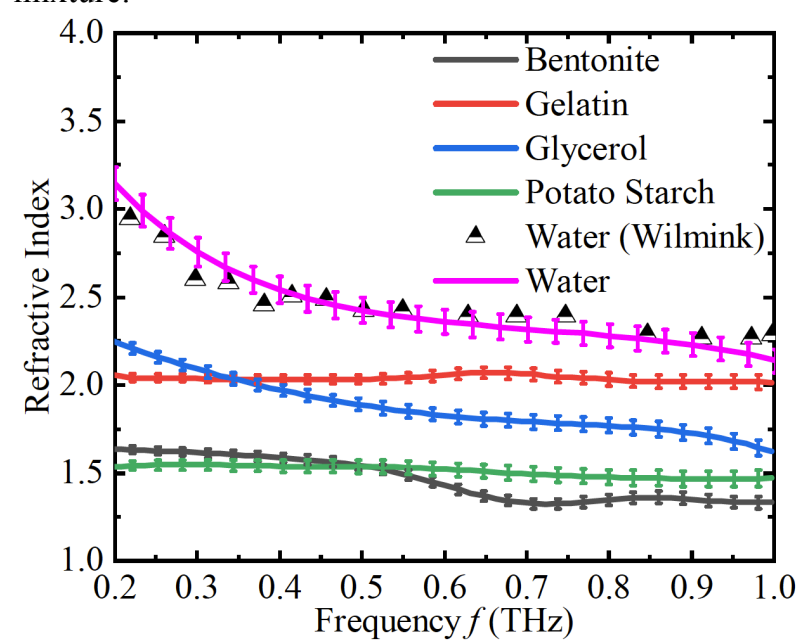

(a)

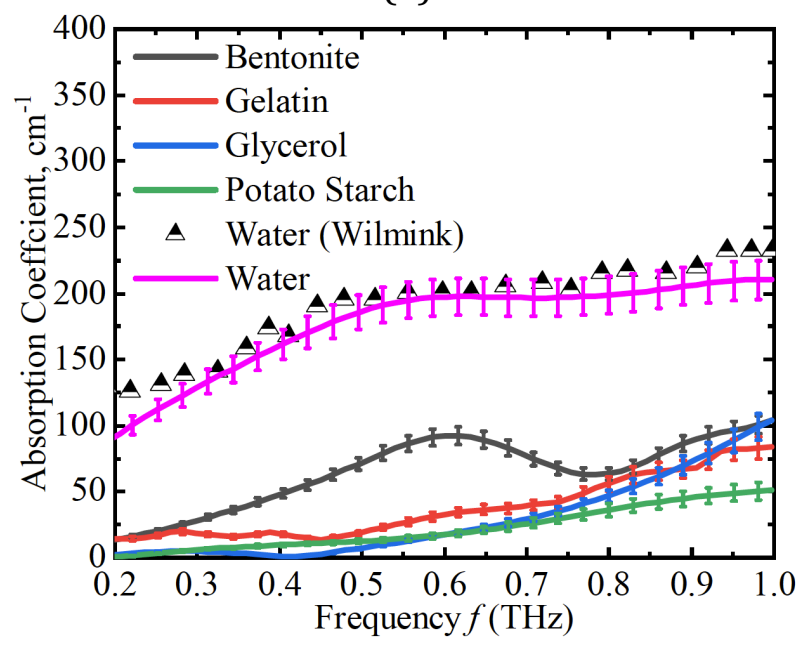

(b)

Fig. 7 The dispersion of (a) refractive index and (b) absorption coefficient of all components in the frequency range of $0.2-1.0 \mathrm{THz}$. The reference data of water are from Ref. [24].
The first EMT theoretical model we decided to use is multi-component CRI model:

$$
\hat{n}_{R}=\sum_{i=1}^{N} f_{i} \hat{n}_{i}
$$

Here $\hat{n}_{R}$ is the complex refractive index of the heterogeneous mixture, $f_{i}$ is the volume fraction for $i$-th inclusion of the dispersed component, $\hat{n}_{i}$ is the complex refractive index of $i$-th inclusion in the heterogeneous mixture.

The second of considered EMT theoretical model is multi-component LLL model:

$$
\sqrt[3]{\hat{\varepsilon}_{R}}=\sum_{i=1}^{N} f_{i} \sqrt[3]{\hat{\varepsilon}_{i}}
$$

where $f_{i}$ is the volume fraction for $i$-th inclusion of the dispersed component, $\hat{\varepsilon}_{R}$ is the complex permittivity of the heterogeneous mixture, $\hat{\varepsilon}_{i}$ is the complex permittivity of the $i$-th dispersed component. This model describes a biopolymer matrix with low volume fraction values of the components and applicable to mixtures of irregularly shaped particles.

The third one, is the mostly used EMT theoretical model - Bruggeman model, which explicitly considers the influence of the inclusions shape and orientation as well structure anisotropy and is described by the following equation [28]:

$$
\sum_{i=1}^{N} f_{i} \frac{\hat{\varepsilon}_{i}-\left(\hat{\varepsilon}_{R}\right)_{p}}{\left(\hat{\varepsilon}_{R}\right)_{p}+v_{i p}\left[\hat{\varepsilon}_{i}-\left(\hat{\varepsilon}_{R}\right)_{p}\right]}=0,
$$

where $\hat{\varepsilon}_{R}$ is the complex permittivity of the heterogeneous mixture, $f_{i}$ is the volume fraction for $i$-th inclusion of the dispersed component, $\hat{\varepsilon}_{i}$ is the complex permittivity of the $i$-th dispersed component, $v_{i p}$ is the depolarization coefficient for the $i$-th inclusion and $p$-th principal axis, $\left(\hat{\varepsilon}_{R}\right)_{p}$ principal values (diagonal elements) of $\hat{\varepsilon}_{R}$.

For the iterative method proposed in Ref. [27], the complex permittivity of two-component (gelatin-inwater) $\hat{\varepsilon}_{R_{1}}$ is calculated first by the Eq. (9) for the spherical particles. Further, the obtained value $\hat{\varepsilon}_{R_{1}}$ is equated to the complex permittivity of matrix inside which the third component (starch) is considered using the Bruggeman equation for the lamellar (disk) particles. By repeating this iteration procedure, first put glycerol (spherical particles) into account, then add bentonite using the formula for disk particles, the complex dielectric constant $\hat{\varepsilon}_{R}$ of the five component mixture can be estimated. The order of components during the iteration was taken in accordance with the order of sample preparation. 


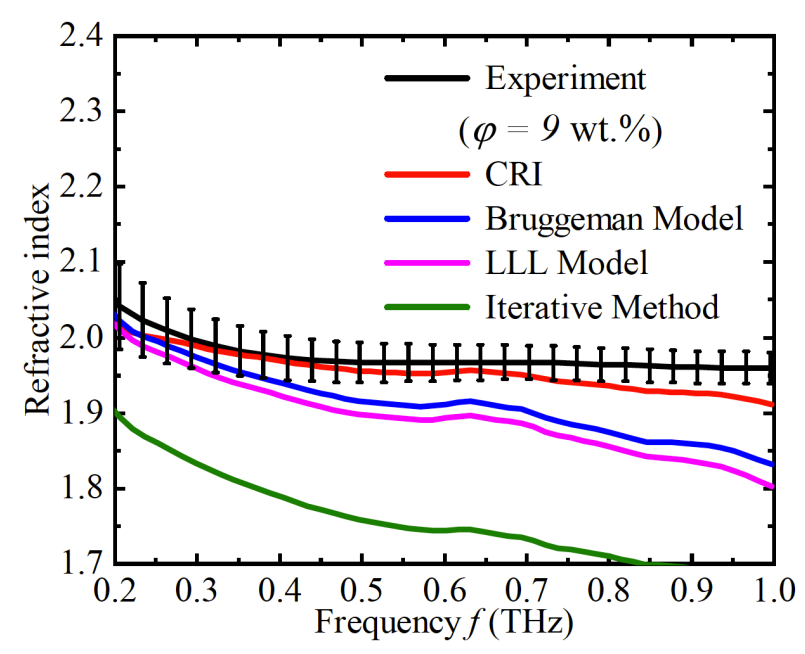

(a)

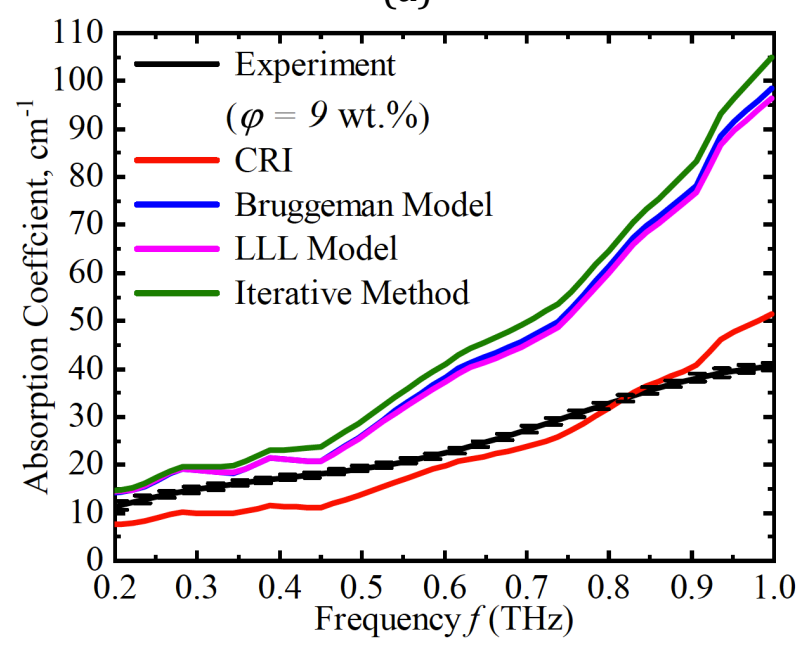

(b)

Fig. 8 Numerical simulation results of the dispersion of (a) refractive index and (b) absorption coefficient of the biopolymers at $\varphi=9 \mathrm{wt} . \%$ in the frequency range of $0.2-1.0 \mathrm{THz}$.

Fig. 8 shows that the refractive index and the absorption coefficient of the biopolymer with the thickness of $306 \mu \mathrm{m}$ is closer to the estimated $\mathrm{THz}$ optical properties obtained using CRI model.

\section{Discussion}

The visual analysis of the biopolymer morphology using optical microscopy (Fig. 2) shows that the biopolymers have a heterogeneous structure with phase separation of the components (see Section 3.1). The observed effect is a consequence caused by the shear stresses of the gelatin and starch solutions at the drying temperature of the gel-forming solution [29]. We previously found [30] that when the biopolymer solution is thermally dried and the molecular mobility of gelatin's polymer chains is higher than that of starch in solution, which leads to the coalescence of the minor phase drops of the starch solution.

In addition to the phases of the biopolymer matrix, the morphology of the biopolymers shows dark regions at their interface, relates mainly to the localized phase of bentonite clay solid particles. Their localization becomes more pronounced with increasing the $\varphi$ value. It is seen that at $\varphi=7$ wt. $\%$ a dense network of filler particles is observed. Such localization of the filler phase is a consequence not only of the relatively high mobility of the polypeptides in the gelatin compared to starch macromolecules, but also of the increased intermolecular interactions between bentonite-waterstarch [31].

A tensile test of the biopolymers was carried out. The result shows that for $\varphi<5$ wt. $\%$, the presence of bentonite particles does not significantly affect the mechanical properties of the material (see Section 3.2). When the concentration of the filler particles rises to $\varphi=5 \mathrm{wt} . \%$, a strong increase in the $\sigma$ value is observed. However, a further increase in $\varphi$ to $9 \mathrm{wt} . \%$ does not lead to significant reinforcing of the polymer matrix, while greatly increasing of $E$ value and, accordingly, the stiffness of the material.

This mechanical behavior of the biopolymers is a consequence of their formed morphology. The introduction of bentonite fillers into the polymer matrix at $\varphi=7 \mathrm{wt} . \%$ leads to the excess of the filler content threshold with the formation of a dense uniform reinforcing network, which brings upmost of the deformation stresses inside the material. A further increase in the filler content leads to the strong localization of its particles and the formation of stress centers, which leads to a decrease in the tensile strength and a strong increase in the stiffness of the material.

The resonance peak appeared in the experiment data in the low terahertz frequency range of $0.3-0.6 \mathrm{THz}$ in Fig. 6 is the resonance caused by starch particles. The resonant frequency can be calculated using the whispering gallery modes approach [32, 33]. As mentioned in the Section 3.1, starch formed a microdomain phase inside of the biopolymer which can be seen from the bright clusters of Fig. 2. The quantity of microdomain phases $N_{s}$ in one cluster should take into account as 4 or 5 . Thus the formula should be written as:

$$
r_{s}=\frac{c}{2 \pi n_{s} f_{r} N_{s}}
$$

where $r_{s}$ is the average radius of the starch particles, $c$ is the light speed, $n_{s}$ is the average refractive index of the starch particles shown in Fig. $7 \mathrm{a}$, and $f_{r}$ is the resonant frequency shown in Fig. 6. If we substitute, according to the known parameters, $N_{s}=4$ or $5, \mathrm{c}=3 \times 10^{8} \mathrm{~m} / \mathrm{s}$, $n_{s}=1.5, f_{r}=0.6 \mathrm{THz}$ into the Eq. (10), then we may accordingly get the possible radius of one starch particles is $r_{s}=13 \pm 2 \mu \mathrm{m}$, which are very close to the average radius of the bright areas in Fig. 2 .

Fig. 9. shows the comparison of the optical properties of the biopolymers with $\varphi=9 \mathrm{wt} . \%$ bentonite and cancerous and healthy oral tissue. The data of oral tissues are from Ref. [6]. It is clear that there are visible diversities between biopolymers and real tissues. Since 
the absorption coefficients of biopolymers are relatively low, $\mathrm{THz}$ radiation may penetrate through the biopolymer with low losses. Thus, diseased region can be easily monitored in $\mathrm{THz}$ frequency range while the biopolymer is being applied as a drug delivery system. In consideration of the obvious differences in refractive indices, a good reflection can be achieved in the interface between biopolymer and tissue, which may give $\mathrm{THz}$ devices a good promise to record noise-less reflection signals in vivo. Furthermore, on account of its good mechanical properties, it can be used as the substrate for tissue surface leveling when an in-vivo measurement needs to be carried out [34]. Moreover, by the fact that changing the content of bentonite doesn't influence on the absorption coefficient of the biopolymer effectively but greatly affects its refractive index, the drug delivery system based on it can be fabricated with different refractive indices for different drugs accordingly, depending on whether the drug layer needs to be taken into account. According to the published researches [35-37], THz-TDS may distinguish the layers of polymeric materials by detecting the reflection of $\mathrm{THz}$ waves and has better resolution than other conventional ultrasonic and optical devices. Thus, the biopolymer may also be used as the potential substrate for in vivo $\mathrm{THz}$ measurements.

\section{Conclusions}

In this work, we obtained a solid-state biopolymer material based on gelatin-starch biopolymer matrix filled with plasticizing glycerol and various loadings of reinforcing bentonite clay particles. The biopolymers were subjected to an analysis of the morphology, mechanical tensile properties and optical properties in the $\mathrm{THz}$ frequency range. Also, the results of optical measurements were analytically described using Complex Refractive Index model as the best theoretical approach.

It is shown that the morphology of biopolymers has a pronounced heterogeneous structure with phase separation of components and strongly depends on the content of bentonite filler. It is found that the introduction of bentonite up to $7 \mathrm{wt} \%$ leads to the formation of a dense reinforcing network in the structure of the material, which entails its strong hardening. With a further increase of bentonite content to $9 \mathrm{wt} . \%$, filler particles are localized in the interfacial region of the material, which leads to the formation of stress centers and reduces the interfacial interaction of the material. These effects lead to reducing the strength and increasing the fragility of the biopolymers.

The THz TDS measurement results show that the refractive index of the biopolymer tends to increase as the bentonite content increases, while the absorption coefficients does not have the same behavior. The $\mathrm{THz}$ optical properties of the biopolymer with the thickness higher than $186 \mu \mathrm{m}$ are more reproducible and stable, and can be accurately described by the CRI model. The absorption troughs appeared at the frequency around
$0.6 \mathrm{THz}$ are caused by the resonant state of starch clusters.

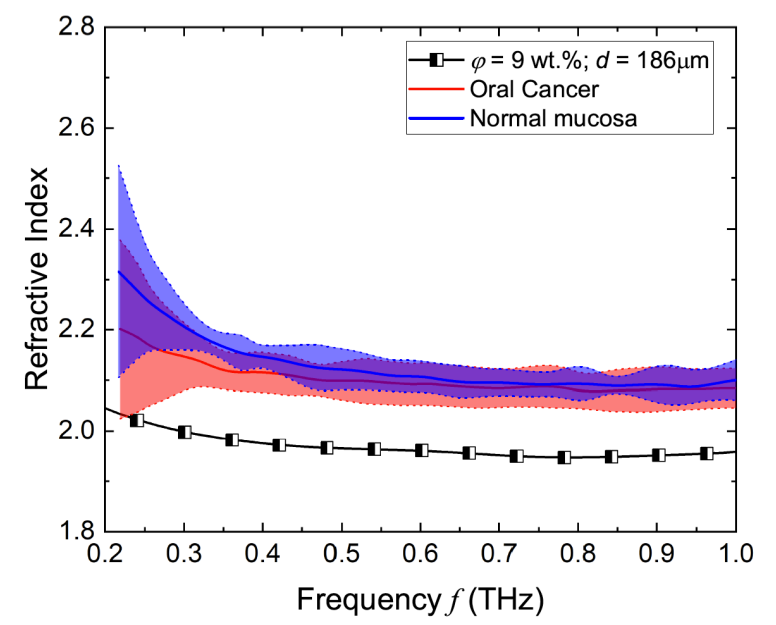

(a)

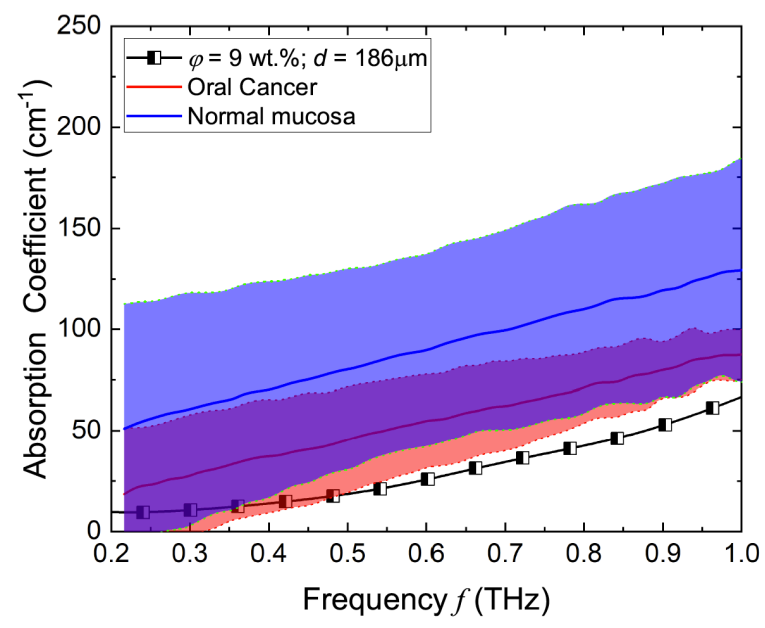

(b)

Fig. 9 Comparison of the (a) refractive index and (b) absorption coefficient of the biopolymers at $\varphi=9 \mathrm{wt} . \%$, cancerous oral tissue [6] and normal mucosa from health oral tissue [6] depending on terahertz frequency.

Due to its relatively lower absorption coefficients, $\mathrm{THz}$ radiation may penetrate through the given biopolymer well. Moreover, from the comparison of $\mathrm{THz}$ optical properties between the fabricated biopolymer and oral tissues, clear diversities in optical properties can be observed. Such $\mathrm{THz}$ optical properties of the biopolymer allow us to confirm the feasibility of using $\mathrm{THz}$ radiation for cancer assessment during therapies. The result of our work shows that $\mathrm{THz}$ waves should be able to distinguish the buccal drug delivery system (biocomposite), drug layer and oral tissue. The biopolymer may also be used as the potential substrate for in-vivo THz measurements.

\section{Disclosure}

All authors declare that there is no conflict of interests in this paper. 


\section{Acknowledgement}

This research was financially supported by the Government of the Russian Federation (Grant 08-08).

\section{References}

1. A. Y. Pawar, D. D. Sonawane, K. B. Erande, and D. V. Derle, “Terahertz technology and its applications,” Drug invention today 5(2), 157-163 (2013).

2. J.-H. Son, Terahertz biomedical science and technology, CRC Press (2014).

3. C. Yu, S. Fan, Y. Sun, and E. Pickwell-MacPherson, "The potential of terahertz imaging for cancer diagnosis: A review of investigations to date," Quantitative imaging in medicine and surgery 2, 33-45 (2012).

4. R. Grigorev, A. Kuzikova, P. Demchenko, A. Senyuk, A. Svechkova, A. Khamid, A. Zakharenko, and M. Khodzitskiy, "Investigation of fresh gastric normal and cancer tissues using terahertz time-domain spectroscopy," Materials 13(1), 85 (2020).

5. M. H. Arbab, D. P. Winebrenner, T. C. Dickey, A. Chen, M. B. Klein, and P. D. Mourad, “Terahertz spectroscopy for the assessment of burn injuries in vivo," Journal of Biomedical Optics 18(7), 077004 (2013).

6. Y. C. Sim, J. Y. Park, K.-M. Ahn, C. Park, and J.-H. Son, "Terahertz imaging of excised oral cancer at frozen temperature," Biomedical Optics Express 4(8), 1413-1421 (2013).

7. Y. B. Ji, E. S. Lee, S.-H. Kim, J.-H. Son, and T.-I. Jeon, "A miniaturized fiber-coupled terahertz endoscope system," Optics Express 17(19), 17082-17087 (2009).

8. G. Calixto, J. Bernegossi, B. Fonseca-Santos, and M. Chorilli, "Nanotechnology-based drug delivery systems for treatment of oral cancer: a review," International journal of nanomedicine 9, 3719 (2014).

9. C. Dianzani, G. P. Zara, G. Maina, P. Pettazzoni, S. Pizzimenti, F. Rossi, C. L. Gigliotti, E. S. Ciamporcero, M. Daga, and G. Barrera, "Drug delivery nanoparticles in skin cancers," BioMed research international 2014, 895986 (2014).

10. J. H. Han, Innovations in Food Packaging, Elsevier B.V. (2005).

11. D. Verma, E. Fortunati, Biopolymer processing and its composites, Elsevier Ltd (2019).

12. O. Moreno, À. Gil, L. Atarés, and A. Chiralt, "Active starch-gelatin films for shelf-life extension of marinated salmon," LWT - Food Science and Technology 84, 189-195 (2017).

13. H. Chen, M. Yang, Z. Shan, S. Mansouri, B. K. May, X. Chen, H. Chen, and M. W. Woo, "On spray drying of oxidized corn starch cross-linked gelatin microcapsules for drug release,” Materials Science and Engineering: C 74, 493-500 (2017).

14. H. Chen, Z. H. Shan, M. W. Woo, and X. D. Chen, "Preparation and characteristic of gelatine/oxidized corn starch and gelatin/corn starch blend microspheres," International Journal of Biological Macromolecules 94, 326-334 (20170.

15. N. Zhang, H. Liu, L. Yu, X. Liu, L. Zhang, L. Chen, and R. Shanks, "Developing gelatin-starch blends for use as capsule materials," Carbohydrate Polymers 92(1), 455-461 (2013).

16. O. Moreno, L. Atarés, A. Chiralt, M. C. Cruz-Romero, and J. Kerry, "Starch-gelatin antimicrobial packaging materials to extend the shelf life of chicken breast fillets," LWT 97, 483-490 (2018).

17. S. Wannaphatchaiyong, P. W. S. Heng, J. Suksaeree, P. Boonme, and W. Pichayakorn, "Lidocaine loaded gelatin/gelatinized tapioca starch films for buccal delivery and the irritancy evaluation using chick chorioallantoic membrane," Saudi Pharmaceutical Journal 27(8), 1085-1095 (2019).

18. V. A. d. S. Garcia, J. G. Borges, V. B. V. Maciel, M. R. Mazalli, J. d. G. Lapa-Guimaraes, F. M. Vanin, and R. A. de Carvalho, "Gelatin/starch orally disintegrating films as a promising system for vitamin C delivery," Food Hydrocolloids 79, 127-135 (2018).

19. J. Vázquez-Cabo, P. Chamorro-Posada, F. J. Fraile-Peláez, Ó. Rubiños-López, J. M. López-Santos, and P. MartínRamos, "Windowing of $\mathrm{THz}$ time-domain spectroscopy signals: A study based on lactose," Optics Communications 366, 386-396 (2016).

20. S. L. Dexheimer, Terahertz spectroscopy: principles and applications, CRC press (2007).

21. D. Swinehart, "The Beer-Lambert law," Journal of Chemical Education 39(7), 333 (1962).

22. M. Naftaly, R. E. Miles, "Terahertz time-domain spectroscopy for material characterization," Proceedings of the IEEE 95(8), 1658-1665 (2007).

23. M. Naftaly, Terahertz metrology, Artech House (2015).

24. G. J. Wilmink, B. L. Ibey, B. D. Rivest, J. E. Grundt, W. P. Roach, T. D. Tongue, B. J. Schulkin, N. Laman, X. G. Peralta, C. C. Roth, C. Z. Cerna, B. D. Rivest, J. E. Grundt, and W. P. Roach, "Development of a compact terahertz time-domain spectrometer for the measurement of the optical properties of biological tissues," Journal of Biomedical Optics 16(4), 047006 (2011). 
25. M. Scheller, C. Jansen, and M. Koch, "Applications of effective medium theories in the terahertz regime," Chapter in Recent Optical and Photonic Technologies, InTech Rijeka, Croatia, 231-250 (2010).

26. V. A. Markel, "Introduction to the Maxwell Garnett approximation: tutorial," Journal of the Optical Society of America A 33(7), 1244-1256 (2016).

27. R. Nazarov, T. Zhang, M. Khodzitsky, and P. Demchenko, "Comparative study of quantitative methods to determine component concentration for water-free biotissue phantom," SPIE Proceedings 11075, 110750Y (2019).

28. D. Schmidt, M. Schubert, "Anisotropic bruggeman effective medium approaches for slanted columnar thin films," Journal of Applied Physics 114(8), 083510 (2013).

29. H. Firoozmand, B. S. Murray, and E. Dickinson, "Microstructure and rheology of phaseseparated gels of gelatin + oxidized starch," Food Hydrocolloids 23(4), 1081-1088 (2009).

30. A. Podshivalov, M. Zakharova, E. Glazacheva, and M. Uspenskaya, "Gelatin/potato starch edible biocomposite films: Correlation between morphology and physical properties," Carbohydrate Polymers 157, 1162-1172 (2017).

31. F. Xie, E. Pollet, P. J. Halley, and L. Avérous, "Starch-based nano-biocomposites," Progress in Polymer Science 38(10-11), 1590-1628 (2013).

32. V. Klimov, Nanoplasmonics, Pan Stanford (2014).

33. G. C. Righini, Y. Dumeige, P. Feron, M. Ferrari, G. Nunzi Conti, D. Ristic, and S. Soria, "Whispering gallery mode microresonators: fundamentals and applications," La Rivista del Nuovo Cimento 34, 435-488 (2011).

34. K. I. Zaytsev, A. A. Gavdush, N. V. Chernomyrdin, and S. O. Yurchenko, "Highly accurate in vivo terahertz spectroscopy of healthy skin: Variation of refractive index and absorption coefficient along the human body," IEEE Transactions on Terahertz science and Technology 5(5), 817-827 (2015).

35. S. Wietzke, C. Jansen, M. Reuter, T. Jung, D. Kraft, S. Chatterjee, B. M. Fischer, and M. Koch, "Terahertz spectroscopy on polymers: A review of morphological studies," Journal of Molecular Structure 1006(1-3), 41-51 (2011).

36. P. Lopato, T. Chady, "Terahertz detection and identification of defects in layered polymer composites and composite coatings," Nondestructive Testing and Evaluation 28(1), 28-43 (2013).

37. A. Redo-Sanchez, B. Heshmat, A. Aghasi, S. Naqvi, M. Zhang, J. Romberg, and R. Raskar, "Terahertz time-gated spectral imaging for content extraction through layered structures," Nature communications 7(1), 1-7 (2016). 